\title{
Diversity in Diffusion-Based Molecular Communication Channel with Drift
}

\author{
Derya Malak Hamideh Ramezani Murat Kocaoglu Ozgur B. Akan \\ Next-generation and Wireless Communications Laboratory \\ Department of Electrical and Electronics Engineering \\ Koc University, Istanbul, Turkey \\ Email:\{dmalak,hramezani13,mkocaoglu,akan\}@ku.edu.tr
}

\begin{abstract}
We utilize the well known Additive Inverse Gaussian Noise (AIGN) communication channel to investigate the effect of diversity in diffusion-based molecular communication with drift, where the transmitter releases different types of molecules to the fluid medium by encoding the information onto the release time and type of molecules. The fluid channel imposes extra delay on the communication, and the receiver decodes the encoded information by solely utilizing the molecular arrival times. In this paper, simple receiver models based on maximum likelihood estimation (MLE) are investigated. Furthermore, upper and lower bounds on the capacity of AIGN communication channel with molecular diversity are derived.
\end{abstract}

Index Terms-Molecular communication, Diffusion, Drift, AIGN channel, Channel capacity, Receiver model, Maximum likelihood estimation

\section{INTRODUCTION}

AIGN channel model is widely used in modeling the delay caused by the drift of the fluid media in molecular communication [1]-[4]. In this paper, we focus on the information encoding on the transmission time and type of the molecules by using the AIGN channel with molecular diversity.

In our model, the transmitter is capable of releasing a diversity of molecules according to a predefined delay distribution. We also assume that there is an abundance of molecules inside the transmitter. The channel is a fluid channel with a positive drift for all the molecule types. Hence, the channel delay can be modeled by the AIGN distribution [2]. Simple receivers, which are capable of storing the timing information of the molecules are presented. The receivers are unable to recognize the molecules identities directly. Instead, they categorize the received molecules based on their arrival times.

For future practical applications of molecular communication [5], [6], transmitting information through the timing and type of the released molecules will be a more realistic model compared to the one in which the information is solely encoded in the molecules' release time. On the other hand, the ability of detecting the molecule type directly complicates the receiver. Thus, we focus on simple receivers which can decode transmitted message and detect the molecule types based on the molecules' arrival time statistics. We also provide the upper and lower capacity bounds for the AIGN communication channel with molecular diversity. Our objective is to point out the rate improvement by using a variety of molecules, compared to the AIGN channel with a single molecule type.
The remainder of this paper is organized as follows. In Section II, we give a model for the AIGN communication channel with molecular diversity. In Section III, we provide simple receiver models to decode the transmitted message by the AIGN channel with low design complexity. In Section IV, to characterize the achievable performance of the AIGN communication channel, we provide the upper and lower bounds on the capacity of the molecular channel based on the receiver characteristics. Then, in Section V, we compare the derived capacity bounds for AIGN channel with molecular diversity. We also evaluate the performance of the proposed receivers in detecting type of molecule and estimating the information encoded in the release time of molecules. Finally, we conclude the paper in Section VI.

\section{Aign Channel With Molecular Diversity}

A molecular communication system based on the release and transmission of the signaling molecules of the same type is analyzed in [2]. Furthermore, an information theoretical model for the additive inverse Gaussian noise (AIGN) channel is derived in the same work. Here, we aim to extend this model to a multi-molecular type communication system. Hence, we consider the AIGN channel with molecular diversity. We aim to observe how the diversity of the transmitted molecules affects the communication performance in fluid medium.

\section{A. Transmitter Model}

The transmitter first determines the molecule type to be released. The molecule type is denoted by a random variable $T$. We assume that there are $M$ types of molecules with each having the release probability of

$$
\operatorname{Pr}(T=i)=\alpha_{i}, \quad i \in\{1, \ldots, M\},
$$

where $\alpha_{i}$ denotes the release probability of molecule type $i$.

The information that the transmitter wants to communicate with the selected molecule type is denoted by random variable $X$. We assume the general case that distribution of $X$ depends on type of the selected molecule. The transmitter encodes this information in the release time of molecules, denoted by random variable $R$. After determining the molecule type and release time, a set of $m$ molecules is released to the fluid medium based on the following scenarios: 
- All the molecules in a set are released simultaneously. In this case, we select $R=X$.

- Molecules of a set are released with different release times. In this case, the parameters of the distribution of $R$ are defined based on $X$.

This transmission process could contribute to design future nanoscale communication techniques for generating possible biological actions externally or artificially. For instance, each molecule type can refer to a distinct action requested from the receiver, and their release time can determine the time variation of the requested action from the receiver.

\section{B. AIGN Channel Model for Fluid Media}

We assume that the molecules propagate independently and the fluid media has a positive drift velocity $v$ and diffusion coefficient $d_{i}$ for each type of molecules, i.e, $i \in\{1, \ldots, M\}$ [7], [8]. We also assume that released molecules propagate by Brownian motion toward receiver, hence the Wiener process can be used to find the pdf of position of $j$ th particle of type $i$, i.e., $w_{i j}$, at time $t>0$ [9] as

$$
\begin{array}{r}
f_{W}\left(w_{i j} ; t\right)=\frac{1}{\sqrt{2 \pi \sigma_{i}^{2} t}} \exp \left(-\frac{\left(w_{i j}-v t\right)^{2}}{2 \sigma_{i}^{2} t}\right), \\
\forall i \in\{1, \ldots, M\} \text { and } \forall j \in\{1, \ldots, m\}
\end{array}
$$

where $\sigma_{i}^{2}=d_{i} / 2$ is the variance of the Wiener process. Assuming that the receiver acts as a perfectly absorbing boundary, we are only concerned with the first arrival time $N$ at the boundary. The same as [2], [10], we assume one dimensional propagation where the transmitter is located at the origin, hence each molecule has initial condition $W(t)=0$. We also assume that the receiver is located at position $l>0$. Hence, the first arrival time is given by

$$
N=\min \{t: W(t)=l\} .
$$

$v>0$ is the necessary condition for the particle to arrive at the receiving boundary. Hence, we assume positive drift so that the pdf of $N$, denoted by $f_{N}(n)$, is given by [11] as

$$
f_{N}(n)= \begin{cases}\sqrt{\frac{\lambda_{i}}{2 \pi n^{3}}} \exp \left(-\frac{\lambda_{i}(n-\mu)^{2}}{2 \mu^{2} n}\right), & n>0 \\ 0, & n \leq 0\end{cases}
$$

which is the IG distribution with mean $\mu=l / v$ and shape parameter $\lambda_{i}=l^{2} / \sigma_{i}^{2}$, i.e., $N \sim \operatorname{IG}\left(\mu, \lambda_{i}\right)$.

Consider the arrival of molecules of set $k$. At time $y_{k j}=$ $r_{k j}+n_{k j}$, the $j$ th molecule released at time $r_{k j}$ arrives at the receiver, where $n_{k j}$ is the first arrival time in the Wiener process for $j$ th molecule of set $k$. The channel is affected by additive noise, in the form of the IG distributed random propagation time $N$. Hence, it is an AIGN channel.

\section{Receiver Model}

The proposed receiver model in [2] is capable of detecting the distinct signaling molecules. Hence, channel uses can take place at any time, or even at the same time, i.e., the channel usages are orthogonal. However, we assume a simpler receiver model such that the receiver is able to reconstruct the transmitted signal using the arrival times of the molecules and does not have the capability of categorizing the molecules according to their types. Since the signaling molecules are indistinguishable, the transmitter must wait until all $m$ molecules have arrived before a new channel use can begin. In this case, the channel uses are not orthogonal. The details of our proposed receivers are explained in the following section.

\section{RECEIVER DESIGN FOR AIGN CHANNEL}

In [2], receiver models are provided for the AIGN channel, which recover the transmitted message (transmission time) from the arrival times of the molecules. Both the ML estimator and the ML detector, and an error probability analysis for the ML detection are provided in this work. In this paper, we extend this model to AIGN channel with molecular diversity. In the proposed model, the receiver determines the type of the molecule based on the statistics of the received distributions, it also estimates the encoded messages in release time. Before describing the receiver structure, we mention a property of IG distribution, which is used in this paper.

Property 1: Let $N_{i} \sim I G\left(\mu_{i}, \lambda_{i}\right), \forall i \in\{1, \ldots, n\}$, be $n$ not necessarily independent random variables, and $N=\sum_{i=1}^{n} c_{i} N_{i}$ for $c_{i}>0$. If constant $\nu$ exists such that $\frac{\lambda_{i}}{c_{i} \mu_{i}^{2}}=\nu, \forall i \in$ $\{1, \ldots, n\}$, then $N \sim I G\left(\sum_{i=1}^{n} c_{i} \mu_{i}, \nu\left(\sum_{i=1}^{n} c_{i} \mu_{i}\right)^{2}\right)[11]$.

In the following, we propose the receiver design for different scenarios described for transmitter.

\section{A. Simultaneous Release of Molecules in a Set}

In this scenario, the transmitter releases a set of $m$ molecules of the same type simultaneously in each channel usage. The receiver collects the arrival time of these molecules, then decides about the type and transmitting time of the set. Assume that the receiver is receiving molecules of $k$ th set, the pdf of observing channel output $Y=y_{k j}$ given channel input $X=x_{k}$ and type of molecule $T=i$ is

$$
\begin{aligned}
& f_{Y \mid X, T}\left(y_{k j} \mid x_{k}, i\right)= \\
& \left\{\begin{array}{ll}
\sqrt{\frac{\lambda_{i}}{2 \pi\left(y_{k j}-x_{k}\right)^{3}}} \exp \left(-\frac{\lambda_{i}\left(y_{k j}-x_{k}-\mu^{2}\right)}{2 \mu^{2}\left(y_{k j}-x_{k}\right)}\right), & y_{k j}>x_{k} \\
0, & y_{k j} \leq x_{k}
\end{array} .\right.
\end{aligned}
$$

Since the distribution of the arrival time of the molecules depends on their type based on (5), we can use statistics of the received distribution to estimate the type of the molecule. The proposed receiver for this section determines the type of the molecule first. Then, it estimates the release time of molecules in a set based on their arrival time.

1) Molecule Type Detection: Since the channel inputs and the noises are independent, the output mean and variance for the $k$ th set containing molecules of type $i$ becomes

$$
\begin{aligned}
& \mathrm{E}[Y \mid T=i]=x_{k}+\mu, \\
& \operatorname{Var}[Y \mid T=i]=\operatorname{Var}[X]+\operatorname{Var}[N \mid T=i]=\frac{\mu^{3}}{\lambda_{i}} .
\end{aligned}
$$


Based on (6), the average of molecules receive time depends on their release time, however, the variance only depends on the type of the molecules. Hence, we design a receiver based on the second moments of the molecular arrival distributions to detect the type of the molecule. In this setting, the condition for the optimal detection is to select a molecule type that minimizes the difference between the calculated and expected values for second moments of the distribution. Hence, we define the following optimization problem to detect $T$.

$$
\hat{T}=\underset{i}{\operatorname{argmin}}\left(\left(\frac{1}{m} \sum_{j=1}^{m} y_{k j}^{2}-\left(\frac{1}{m} \sum_{j=1}^{m} y_{k j}\right)^{2}\right)-\left(\frac{\mu^{3}}{\lambda_{i}}\right)\right)^{2},
$$

2) Transmission Time Estimation: The receiver observes $Y=y_{k j}, j \in\{1, \ldots, m\}$ and computes an estimate of $X$, i.e., $\hat{X}$, when type of the molecule is detected based on (7), i.e., $\hat{T}=i$ is known. Since the propagation of different molecules in each set is assumed to be independent, the ML estimator of $X$, denoted by $\hat{X}_{M L}$, is given by

$$
\hat{X}_{M L}=\arg \max _{x} \prod_{j=1}^{m} f_{Y \mid X, T}\left(y_{k j} \mid x, i\right),
$$

where $f_{Y \mid X, T}\left(y_{k j} \mid x, i\right)$ is defined in (5).

Since the ML estimator given in (8) does not have a closed form solution, a simpler estimator based on the average of the received signals, which is called $Z$, can be used [2]. Hence, the receiver calculates $z_{k}=\frac{1}{m} \sum_{j=1}^{m} y_{k j}$ for $k$ th set.

Since the distribution of arrival time of each molecule is $I G\left(E[X]+\mu, \lambda_{i}\right)$ and based on Property 1 , the distribution of random variable $Z$ is $I G\left(E[X]+\mu, m \lambda_{i}\right)$. Hence, using the average arrival time of $m$ molecules is the same as dividing the variance of the received signal by $m$ in the AIGN channel without molecular diversity.

The ML estimator of $X$ based on $Z$ can be calculated as

$$
\hat{X}_{M L}=z_{k}+\frac{\mu^{2}}{m \lambda_{i}}\left(\frac{3}{2}-\sqrt{\frac{9}{4}+\frac{m^{2} \lambda_{i}^{2}}{\mu^{2}}}\right) .
$$

\section{B. Separate Release of Molecules in a Set}

In this scenario, we assume that the parameters of noise for each type of molecules, i.e., $\mu$ and $\lambda_{i}, \forall i \in\{1, \ldots, M\}$, are known in receiver and transmitter by training as described in [2]. Considering $X=x_{k}$ and $T=i$ for $k$ th set, we select the distribution of molecules' release time based on $X$ as

$$
R \sim I G\left(\mu x_{k}, \lambda_{i} x_{k}^{2}\right)
$$

Since $Y=R+N$ and based on Property 1, the distribution of the molecules' receive time when $X$ and $T$ are known become

$$
Y \mid X, T \sim I G\left(\mu\left(1+x_{k}\right), \lambda_{i}\left(1+x_{k}\right)^{2}\right),
$$

where $\lambda_{i}=\frac{2 l^{2}}{d_{i}}$ and the receiver needs to find discrete parameter $d_{i}$ and continuous variable $x_{k}$ based on the molecules' arrival time. For this aim, we propose two receivers as follows.
1) Full Search-Based Receiver (FS-Receiver): The possible values of $d_{i}, \forall i \in\{1, \ldots, M\}$ are known at the receiver. If a few type of molecules are used in the communication, i.e., $M$ is a small number, the receiver can search over different values of $d_{i}$ and find the one that maximizes the log-likelihood function. In this part, we use the same approach to propose the receiver structure. To estimate the optimal value of $X$ according to each $d_{i}$, we assume $d_{i}$ as a constant and solve the ML estimation problem and reach

$$
\hat{X}_{M L}=\frac{\frac{1}{\mu}+\sqrt{\left(\frac{1}{\mu}\right)^{2}+\frac{2 d_{i}}{m l^{2}} \sum_{j=1}^{m} \frac{1}{y_{k j}}}}{\frac{2}{m} \sum_{j=1}^{m} \frac{1}{y_{k j}}}-1 .
$$

In the receiver, the optimal value of $X$ for each $d_{i}$, called $\hat{x}_{i, M L}$, is calculated based on (12). Then, the pair of $\left(i, \hat{x}_{i, M L}\right)$ that maximizes the likelihood function is selected as detected type and estimated value for $X$ by

$$
\hat{T}_{F S}, \hat{X}_{F S}=\arg \max _{i, \hat{x}_{i, M L}} \prod_{j=1}^{m} f_{Y \mid X, T}\left(y_{k j} \mid x, i\right) .
$$

2) Maximum Likelihood-Based Receiver (ML-Receiver): Increasing $M$ increases the complexity of the FS-Receiver, hence we propose a simpler receiver in this section. First, we assume that both $d_{i}$ and $x_{k}$ are continuous random variables and solve the ML estimation problem as

$$
\begin{aligned}
\hat{X}_{M L} & =\frac{1}{m \mu} \sum_{i=1}^{m} y_{k j}-1, \\
\hat{d}_{M L} & =\frac{2 l^{2}}{m \mu^{2}} \sum_{i=1}^{m} y_{k j}\left(\sum_{i=1}^{m} \frac{1}{m} y_{k j} \sum_{i=1}^{m} \frac{1}{m y_{k j}}-1\right) .
\end{aligned}
$$

Next step is selecting the type of the molecule. One approach can be selecting type according to the nearest $d_{i}, \forall i \in$ $\{1, \ldots, n\}$ to $\hat{d}_{M L}$. However, it might not lead to the optimum point. Hence, we propose another method. Based on (11), the ratio of variance of $(Y \mid X, T)$ over its mean is

$$
\frac{\operatorname{Var}[Y \mid X, T]}{\mathrm{E}[Y \mid X, T]}=\frac{\frac{\mu^{3}\left(1+x_{k}\right)^{3}}{\lambda_{i}\left(1+x_{k}\right)^{2}}}{\mu\left(1+x_{k}\right)}=\frac{\mu^{2}}{\lambda_{i}}=\frac{d_{i}}{2 v^{2}} .
$$

Since this ratio only depends on $d_{i}$, we use it to detect $T$ by

$$
\hat{T}=\underset{i}{\operatorname{argmin}}\left(\frac{\frac{1}{m} \sum_{j=1}^{m} y_{k j}^{2}-\left(\frac{1}{m} \sum_{j=1}^{m} y_{k j}\right)^{2}}{\frac{1}{m} \sum_{j=1}^{m} y_{k j}}-\frac{1}{2 v^{2}} d_{i}\right)^{2} \text {. }
$$

\section{CAPACITY BOUNDS FOR AIGN CHANNEL WITH MOLECUlAR DiVERSITY}

Capacity bounds for single type molecular AIGN channel are derived in [2]. We will see that if the receiver-side had the capability of distinguishing molecules, for the multi-molecular type AIGN channel, the upper and lower capacity bounds would be the same as single type molecular AIGN. However, we assume that the receiver is only able to decode the transmitted messages via type and release time of molecules through 
the molecule reception times. In this section, we provide the upper and lower bounds for the capacity of the multi-molecular type AIGN channels described in this paper. Moreover, we compare their capacity with the case that receiver is able to detect molecule type directly.

The mutual information between the inputs and the output of the AIGN channel is given by

$$
\begin{aligned}
I(X, T ; Y) & =h(Y)-h(Y \mid X, T)=h(Y)-h(N \mid T) \\
& =h(Y)-\sum_{i=1}^{M} \alpha_{i} h_{I G\left(\mu, \lambda_{i}\right)},
\end{aligned}
$$

where $h_{I G\left(\mu, \lambda_{i}\right)}$ is the differential entropy of the IG distribution defined in [2, Eq. (11)]. The maximum of this mutual information over all possible input distributions gives the capacity of the channel. Since the information is encoded in the release time of the molecules, the constraints on the input, which determine the possible input distributions, are application dependent. Hence, the same as [2], we use the mean constraint for each type of molecules, i.e., $\mathrm{E}[X \mid T=i] \leq m_{x, i}$, to find all possible input distributions. Hence, the capacity of the AIGN channel can be calculated by

$$
C=\max _{\sum_{i=1}^{m} \alpha_{i}=1, f(x): \mathrm{E}[X] \leq \sum_{i=1}^{m} \alpha_{i} m_{x, i}} I(X, T ; Y) .
$$

\section{A. Upper Bounds for AIGN Channel with Molecular Diversity}

1) Simultaneous Release of Molecules in a Set: In this section, we consider that all $m$ molecules are released simultaneously and provide upper bounds for the AIGN channel with molecular diversity based on whether the receiver could recognize the molecule types or not.

- Molecules are distinguishable at the receiver: As described in [2, Eq. (18)], in this case, the individual capacity of each channel would be bounded by

$$
C_{i} \leq \log \left(\left(m_{x, i}+\mu\right) e\right)-h_{I G\left(\mu, \lambda_{i}\right)} .
$$

Since the receiver knows the molecule type it receives, the capacity of the AIGN molecular channel would be upper bounded by $C_{U 1}=\max _{\sum \alpha_{i}=1} \sum_{i=1}^{M} \alpha_{i} C_{i}$.

Since $0 \leq \alpha_{i} \leq 1, \forall i$, this upper bound can be derived by selecting $\alpha_{u_{1}}=1$ and $\alpha_{i}=0, \forall i \neq u_{1}$, where

$$
u_{1}=\underset{i \in\{1, \ldots, M\}}{\arg \max } \log \left(\left(m_{x, i}+\mu\right) e\right)-h_{I G\left(\mu, \lambda_{i}\right)},
$$

then $C_{U 1}$ simplifies to

$$
C_{U 1}=\max _{i \in\{1, \ldots, M\}} \log \left(\left(m_{x, i}+\mu\right) e\right)-h_{I G\left(\mu, \lambda_{i}\right)} .
$$

By comparing (18) and (20), we see that the capacity of AIGN channel with molecular diversity when molecules are distinguishable at receiver is the same as the capacity of AIGN channel without molecular diversity, where the utilized molecule type is selected based on (19).

- Molecules are not distinguishable at the receiver: In this case, the average channel output is

$$
\mathrm{E}[Y]=E[E[Y \mid T=i]] \leq \sum_{i=1}^{M} \alpha_{i}\left(m_{x, i}+\mu\right) .
$$

Hence, $h(Y) \leq \log \left(\sum_{i=1}^{M} \alpha_{i}\left(m_{x, i}+\mu\right) e\right)$ with equality if $Y$ has exponential distribution with $\mathrm{E}[Y]=$ $\sum_{i=1}^{M} \alpha_{i}\left(m_{x, i}+\mu\right)$ [2]. Therefore, based on (16), (17), the capacity upper bound $C_{U 2}$ can be calculated as

$$
\begin{aligned}
& C_{U 2}= \\
& \quad \max _{\sum \alpha_{i}=1}\left[\log \left(\sum_{i=1}^{M} \alpha_{i}\left(m_{x, i}+\mu\right) e\right)-\sum_{i=1}^{M} \alpha_{i} h_{I G\left(\mu, \lambda_{i}\right)}\right] .
\end{aligned}
$$

If we can find a pdf for $X: \mathrm{E}[X \mid T=i] \leq m_{x, i}$ that causes the exponential distribution for $Y$, then the expressions in (20) and (22) would be the true capacities of AIGN channel for mean constrain when molecules are distinguishable and are not distinguishable, respectively. Based on [2], the $Y$ distribution tends to exponential by selecting exponential distribution for $X$ when $v \rightarrow \infty$. Hence, these upper bounds are achievable in high velocity, however, as shown in [2] they are not achievable at low velocities.

Since $\log (\cdot)$ is concave, $\log \left(\sum_{i=1}^{M} \alpha_{i}\left(m_{x, i}+\mu\right) e\right) \geq$ $\sum_{i=1}^{M} \alpha_{i} \log \left(\left(m_{x, i}+\mu\right) e\right)$. Hence, $C_{U 2} \geq C_{U 1}$, i.e., the capacity upper bound for the AIGN channel when molecules are not distinguishable at receiver is larger than the AIGN channel when molecules are distinguishable. The reason is the fact that the mutual information of the input and output can be written as $I(X ; Y \mid T)=h(Y \mid T)-h(Y \mid X, T)$ when the type of the molecules are know in the receiver. By comparing this mutual information with (16), we see that knowing the type of the molecule decreases the capacity since $h(Y \mid T) \leq h(Y)$.

2) Separate Release of Molecules in a Set: In this case, the release time of $m$ molecules in a set is defined by (10). Hence, based on (11), we have $\mathrm{E}\left[Y \mid X=x_{k}, T=i\right]=\mu\left(1+x_{k}\right)$ and

$$
\begin{aligned}
\mathrm{E}[Y] & =\sum_{i=1}^{M} \alpha_{i} \int f\left(x_{k} \mid T=i\right) \mu\left(1+x_{k}\right) d x_{k} \\
& =\sum_{i=1}^{M} \alpha_{i} \mu(1+\mathrm{E}[X \mid T=i]) \leq \sum_{i=1}^{M} \alpha_{i} \mu\left(1+m_{x, i}\right) .
\end{aligned}
$$

Hence, the entropy of the output $Y$ is upper bounded by

$$
h(Y) \leq \log \left(\sum_{i=1}^{M} \alpha_{i} \mu\left(1+m_{x, i}\right) e\right) .
$$

By applying (23) to (16) and based on (17), the capacity upper bound $C_{U 3}$ is calculated as

$$
\begin{aligned}
& C_{U 3}= \\
& \quad \max _{\sum \alpha_{i}=1}\left[\log \left(\sum_{i=1}^{M} \alpha_{i} \mu\left(1+m_{x, i}\right) e\right)-\sum_{i=1}^{M} \alpha_{i} h_{I G\left(\mu, \lambda_{i}\right)}\right] .
\end{aligned}
$$




\section{B. Lower Bounds for AIGN Channel with Molecular Diversity}

1) Simultaneous Release of Molecules in a Set : A lower bound on the AIGN channel capacity can be obtained by assuming that $(X \mid T=i)$ is IG distributed as follows,

$$
(X \mid T=i) \sim \operatorname{IG}\left(m_{x, i},\left(\lambda_{i} / \mu^{2}\right) m_{x, i}^{2}\right) .
$$

Since $(N \mid T=i) \sim \operatorname{IG}\left(\mu, \lambda_{i}\right)$, and based on Property 1 , the distribution of $(Y \mid T=i)$ is obtained as

$$
(Y \mid T=i) \sim \operatorname{IG}\left(m_{x, i}+\mu,\left(\lambda_{i} / \mu^{2}\right)\left(m_{x, i}+\mu\right)^{2}\right) .
$$

Hence, the entropy of the receiver input can be denoted as

$$
h(Y \mid T)=h_{I G\left(m_{x, i}+\mu,\left(\lambda_{i} / \mu^{2}\right)\left(m_{x, i}+\mu\right)^{2}\right)} .
$$

In the following, we provide two different lower bounds for the AIGN channel with molecular diversity based on whether the receiver could recognize the molecule types or not.

- Molecules are distinguishable at the receiver: If the receiver-side knew the molecule types,

$$
\max _{f_{X}(x): \mathrm{E}[X \mid T=i] \leq m_{x, i}} h(Y) \geq \sum_{i=1}^{M} \alpha_{i} h(Y \mid T) .
$$

Hence, based on (16), (17) and by selecting $\alpha_{l_{1}}=1$ and $\alpha_{i}=0, i \neq l_{1}$ and $\forall i \in\{1, \ldots, M\}$, where

$l_{1}=\underset{i \in\{1, \ldots, M\}}{\arg \max } h_{I G\left(m_{x, i}+\mu,\left(\lambda_{i} / \mu^{2}\right)\left(m_{x, i}+\mu\right)^{2}\right)}-h_{I G\left(\mu, \lambda_{i}\right)}$,

the capacity of the channel would be lower bounded by

$$
C_{L 1}=h_{I G\left(m_{x, l_{1}}+\mu,\left(\lambda_{l_{1}} / \mu^{2}\right)\left(m_{x, l_{1}}+\mu\right)^{2}\right)}-h_{I G\left(\mu, \lambda_{l_{1}}\right)} .
$$

- Molecules are not distinguishable at the receiver: In this case, the entropy of the $Y$ is

$$
h(Y)=h(T)+\sum_{i=1}^{M} \alpha_{i} h(Y \mid T=i)
$$

where, $h(T)=\sum_{i=1}^{M}-\alpha_{i} \log \left(\alpha_{i}\right)$. Hence, the lower bound of the channel capacity is

$$
C_{L 2}=\sum_{i=1}^{M} \alpha_{i}\left(h(Y \mid T=i)-\log \left(\alpha_{i}\right)-h_{I G\left(\mu, \lambda_{i}\right)}\right),
$$

where $C \geq C_{L 2}$. By selecting $\alpha_{l_{1}}=1$ and $\alpha_{i}=0, \forall i \in$ $\{1, \ldots, M\}$ and $i \neq l_{1}, C_{L 2}$ simplifies to $C_{L 2}=C_{L 1}$.

2) Separate Release of Molecules in a Set: A lower bound on the capacity of the AIGN channel in this case can be obtained by assuming $(X \mid T=i)=m_{x, i}$. Hence, entropy of $Y$ can be obtained by (29), where based on (11), $h(Y \mid T=i)$ is given by

$$
h(Y \mid T=i)=h_{I G\left(\mu\left(1+m_{x, i}\right), \lambda_{i}\left(1+m_{x, i}\right)^{2}\right)} .
$$

Based on (16), (17) and by selecting $\alpha_{l_{3}}=1, \alpha_{i}=0, \forall i \in$ $\{1, \ldots, M\}$ and $i \neq l_{3}$, where

$$
l_{3}=\underset{i \in\{1, \ldots, M\}}{\arg \max }\left(h_{I G\left(\mu\left(1+m_{x, i}\right), \lambda_{i}\left(1+m_{x, i}\right)^{2}\right)}-h_{I G\left(\mu, \lambda_{i}\right)}\right),
$$

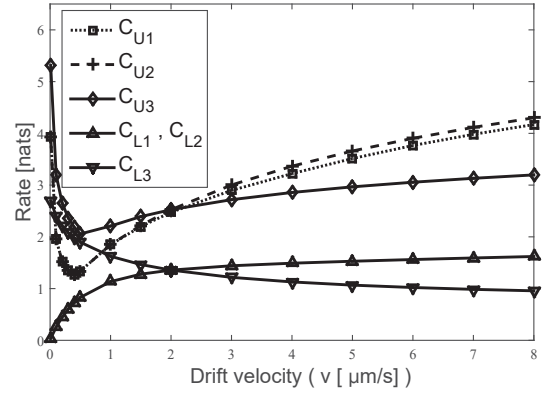

Fig. 1. Upper and lower bounds of capacity as a function of the drift velocity.

a lower bound for the channel capacity can be obtained as

$$
C_{L 3}=h_{I G\left(\mu\left(1+m_{x, l_{3}}\right), \lambda_{l_{3}}\left(1+m_{x, l_{3}}\right)^{2}\right)}-h_{I G\left(\mu, \lambda_{l_{3}}\right)} .
$$

\section{NUMERICAL RESUltS}

By selecting parameters of the system as $M=4, l=2 \mu \mathrm{m}$, $\alpha_{i}=\frac{1}{M}, \forall i \in\{1, \ldots, 4\}, m_{x, i}=\{1,4,2,3\}$, and $d_{i}=$ $\{0.1,1,10,20\} \frac{\mu \mathrm{m}^{2}}{\mathrm{~s}}$, which are in the range of realistic values for diffusion coefficient of molecules [12], we report two types of numerical results in this section as follows.

\section{A. Capacity Bounds}

The capacity bounds for AIGN channel with molecular diversity with respect to the drift velocity $v$, are illustrated in Fig. 1. As explained in Section IV-A1 and depicted in Fig. $1, C_{U 2} \geq C_{U 1}$. Note that their difference depends on the relation between type of the molecules and the mean of information encoded in release time of each type. For instance, by comparing (20) and (22), we see that if the type of the molecule that has minimum $h_{I G\left(\mu, \lambda_{i}\right)}$, has also maximum $m_{x, i}$, then $C_{U 2}$ becomes equal to $C_{U 1}$.

Comparing (22) and (24), we see that $C_{U 3} \geq C_{U 2}$ for $\mu>1$ and $C_{U 3}<C_{U 2}$ for $\mu<1$. Hence, by utilizing $l=2 \mu \mathrm{m}$, we have $C_{U 3} \geq C_{U 2}$ for $v<2 \frac{\mu \mathrm{m}}{\mathrm{s}}$ as depicted in Fig. 1 .

Based on Fig. 1, if $v$ is small, the capacity lower bound $C_{L 3}$ is greater than the upper bound $C_{U 1}$, which is equal to the upper bound capacity of AIGN channel without molecular diversity. Moreover, for high velocities $C_{U 2}$, which is greater than $C_{U 1}$, is achievable as explained in Section IV-A1. Hence, based on amount of $v$, we can select the appropriate transmission scenario for AIGN molecular communication channel with diversity, which yields to greater achievable capacity compared to the AIGN channel without molecular diversity.

\section{B. Detection/Estimation Error}

By assuming Exponential distribution for $(X \mid T=i), \forall i$, the effects of drift velocity, i.e., $v$, and number of molecules in a set, i.e., $m$, on detecting type of the molecule and estimating the encoded information in release time of molecules are depicted in Fig. 2 and Fig. 3, respectively. Increasing $m$ decreases the error, as expected. Furthermore, since increasing the drift velocity decreases the mean and variance of the IG noise, it also decreases the error. 


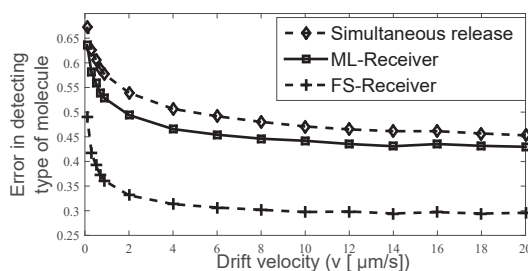

(a) $m=M$

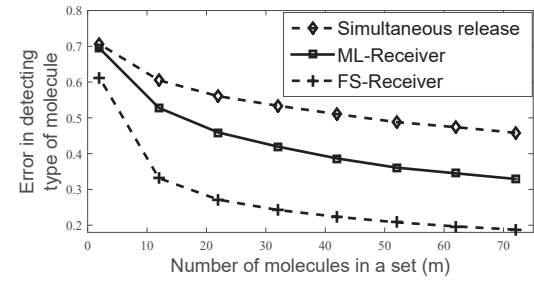

(b) $v=0.1 \frac{\mu m}{\mathrm{~s}}$

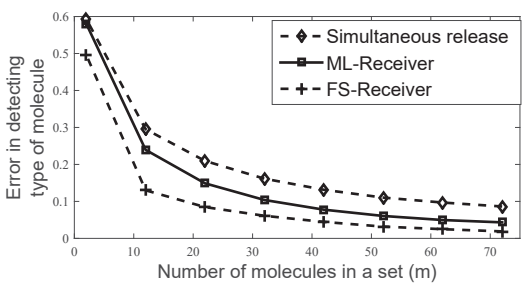

(c) $v=10 \frac{\mu m}{\mathrm{~s}}$

Fig. 2. Effects of the drift velocity, $v$, and number of molecules, $m$, on error in detecting the type of molecules

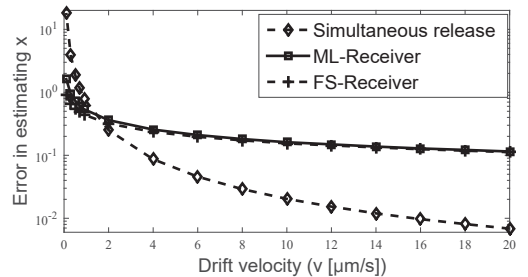

(a) $m=M$

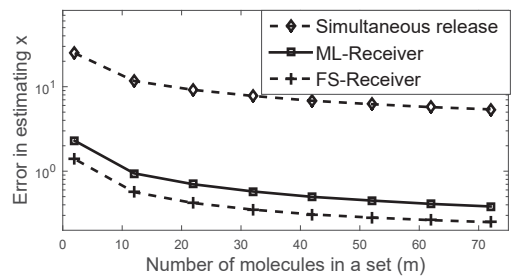

(b) $v=0.1 \frac{\mu m}{\mathrm{~s}}$

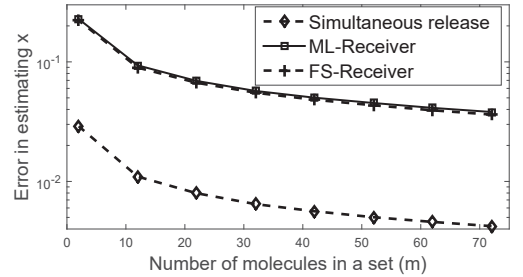

(c) $v=10 \frac{\mu m}{\mathrm{~s}}$

Fig. 3. Effects of the drift velocity, $v$, and number of molecules in a set, $m$, on error in estimating the information encoded on release time of molecules

Based on Fig. 2, using IG distribution for release time of molecules in a set gives lower detection errors compared to simultaneous release. The reason is the fact that in the first one, both IG noise and $R$ have information about type of the molecule, however, only IG noise is used to detect type of the molecule in the second one. On the other hand, the relative performance of these methods for estimating the encoded information in release time depends on the drift velocity as depicted in Fig. 3. The reason is that by increasing $v>l$ the mean and variance of IG noise and IG distributed $R$ decrease such that by selecting $v \rightarrow \infty$ the IG distribution tends to Dirac delta function [13]. Hence, by increasing drift velocity, the effect of the noise on the arrival time of the simultaneously released molecules decreases.

Comparing the receivers proposed for the scenario that molecules in a set are releasing separately, FS-Receiver outperforms $M L$-Receiver, as expected. However, its complexity increases by increasing type of molecules.

\section{CONCLUSION}

In this paper, we considered the AIGN channel with molecular diversity, where the data is encoded in type and release time of molecules. After selecting the type of the molecule, a set of molecules with the defined type are released to the channel with two scenarios (1) all molecules release simultaneously, (2) molecules release separately. We proposed statistical receiver models for these scenarios assuming that receiver is not able to distinguish molecule type directly. Furthermore, we calculated upper and lower bounds for the capacity of AIGN channel with molecular diversity. The analysis is promising for future molecular applications, such as diagnosing different types of molecular signals, and generating the required time varying biological responses according to the molecular diversity, as requested by the transmitter.

\section{ACKNOWLEDGMENT}

This work is a part of the project MINERVA supported by the European Research Council (ERC) under grant ERC-2013CoG \#616922.

\section{REFERENCES}

[1] B. D. Unluturk, D. Malak, and O. B. Akan, "Rate-delay tradeoff with network coding in molecular nanonetworks," IEEE Transactions on Nanotechnology, vol. 12, no. 2, pp. 120-128, 2013.

[2] K. Srinivas, A. W. Eckford, and R. S. Adve, "Molecular communication in fluid media: The additive inverse gaussian noise channel," IEEE Transactions on Information Theory, vol. 58, no. 7, pp. 4678-4692, 2012.

[3] B. D. Unluturk, E. B. Pehlivanoglu, and O. B. Akan, "Molecular channel model with multiple bit carrying molecules," in Communications and Networking (BlackSeaCom), 2013. IEEE, 2013, pp. 79-83.

[4] M. N. Khormuji, "On the capacity of molecular communication over the aign channel," in 45th Annual Conference on Information Sciences and Systems (CISS). IEEE, 2011, pp. 1-4.

[5] I. F. Akyildiz, F. Brunetti, and C. Blázquez, "Nanonetworks: A new communication paradigm," Computer Networks, vol. 52, no. 12, pp. 2260-2279, 2008.

[6] L. P. Giné and I. F. Akyildiz, "Molecular communication options for long range nanonetworks," Computer Networks, vol. 53, no. 16, pp. 2753-2766, 2009.

[7] M. Pierobon and I. F. Akyildiz, "Capacity of a diffusion-based molecular communication system with channel memory and molecular noise," IEEE Tran. on Information Theory, vol. 59, no. 2, pp. 942-954, 2013.

[8] S. Kadloor, R. S. Adve, and A. W. Eckford, "Molecular communication using brownian motion with drift," IEEE Transactions on NanoBioscience, vol. 11, no. 2, pp. 89-99, 2012.

[9] I. Karatzas and S. Shreve, Brownian motion and stochastic calculus. Springer Science \& Business Media, 2012, vol. 113.

[10] A. W. Eckford, K. Srinivas, and R. S. Adve, "The peak constrained additive inverse gaussian noise channel," in International Symposium on Information Theory Proceedings (ISIT). IEEE, 2012, pp. 2973-2977.

[11] R. Chhikara and J. Folks, "The inverse gaussian distribution theory," in Methodology and Application Statistics. Marcel Dekker, 1989.

[12] J. Berthier and P. Silberzan, "Microfluidics for biotechnology; artech house: Boston, 2006."

[13] V. Seshadri, The inverse Gaussian distribution: statistical theory and applications. Springer Science \& Business Media, 2012, vol. 137. 